\title{
ÜBERSICHT DER NEUANGELSÄCHSISCHEN SPRACHDENKMÄLER.
}

$\mathrm{H}_{\text {ickes ist der erste gelehrte gewesen, welcher es versuchte }}$ eine historische grammatik des englischen bis etwa zum jahre $1200 \mathrm{zu}$ schreiben. $\left.{ }^{1}\right)$ Er teilt die sprache vom einfalle der Angelsachsen bis zur genannten zeit in drei perioden: ${ }^{2}$ )

1. Die erste nennt er britannisch-sächsische periode, die sprache lingua Britanno-Saxonica oder auch purior Saxonica.

Von denkmälern führt er daraus nur die bei Älfred erhaltnen zeilen Cädmons an. ${ }^{3}$ )

2. Mit den am ende des 8 . jahrhunderts häufiger werdenden einfällen der Dänen beginnt dann die zweite periode, die dänisch-sächsische und dauert bis zur eroberung durch die Normannen.

In diese zeit fallen also alle damals bekannten angelsächsischen denkmäler.

3. Der dritte zeitabsehnitt ist dann der normanno-dano sächsische.

In dieser periode gehen nach Hickes ${ }^{4}$ ) zwei dialecte neben einander, die lingua Semi-Saxonica, welche dem anglo-säch-

1) Vgl. Hickesii thesaurus linguarum septentrionalium. Oxford 1705. 3 bde.

2) a. a. o. I. pag. 87. (ap. 19.

3) Es sind natürlich die bei Älfred in seiner ïbersetzung des Beda IV, 24 angeführten zeilen gemeint, die beginnen:

$\mathrm{Nu}$ ve sceolon hêrjan heofonrîces veard, metodes mihte and his môdgeponc

wicht die nordhumbrisch abgefassten:

$\mathrm{Nu}$ scylun hergan hefænricæs vard

metudæs mæcti end his modgidanc.

4) Vgl. Hickes cap. 22 p. 134 u. 146.

Beiträge zur geschichte der deutschen sprache. I. 
sisch des zweiten abschnittes und die lingua Anglo-Normannica vel Normanno-Saxonica, welche dem anglo-dannisch entspricht.

Wie Hickes den ausdruck Semi-Saxon verstanden wissen will, sagt er selbst deutlich: Qucmadmodum enim vinum acescens, nondum tamen in acetum conversum, semivinum vel semiacetum dici usu adprobante possit; sic australium nostrorum Saxonum medium illum vel ad medium accedentem sermonem Semi-Saxonicum et, si usus vellet, Semi-Anglicum haud absurde nuncupandum esse judicamus. Auf diese periode folgt bei Hickes dann die englische sprache.

Warton behält die selbe einteilung bei und erklärt vom Semi-Saxon oder Norman-Saxon: ${ }^{5}$ ) it formed a language extremely barbarous, irregular and intractable; and consequently promises no very striking specimens in any species of composition. Its substance was the Danish Saxon, adulterated with french. ${ }^{6}$ )

Grimm erkannte mit scharfem blicke, dass die einteilung von Hickes unhaltbar, doch da er einsah, dass nur jemand, der die ags. mss. vor augen hätte, eine wirklich haltbare aufstellung geben könne, gab er die angelsächsische laut- und formenlehre und schloss daran das mittel- und neuenghische, ${ }^{7}$ ) ohne eine genauere scheidung zu versuchen.

Wright teilt in Anglo-Saxon and Anglo-Norman period ein ${ }^{8}$ ). Bei letzterer finden sich ausser schriftstellern, welche latein

5) Warton, history of English poetry from the close of the 11th. centur.y to the commencement of the $18^{\text {th. }}$ century. London 1840. I.bd. p. $1 \mathrm{u}$. ff. Die erste ausgabe davon erschien 1774.

6) W. schliesst diese periode mit dem tode Heinrichs II. (1189), $\mathrm{Er}$ setzt hinein ausser den werken, welche man gewöhnlich in die zeit von 1400-1250 setzt, (siehe unten) die juingere Marherete, heiligenleben, die weit später anzusetzen sind, the land of Cokaygne, dann volkslieder, wie Sumer is icumen in, Blow northerne wind u. 2, ebenfalls Horn ohild. Vom wirklichen alter dieser lieder hat er gar kein verständnis. Madden hat ihm in anmerkungen seine fehler nachgewiesen und so liest jetzt der lespr. gapze seiten bei Warton, um dann in den apmerkungen zu erfahren, dass alles gelesene durchaus unrichtig ist. Warum setzen so treffliche gelehrte, wie Madden, nicht eine unzeitige pietät gegen Warton bei seite und arbeiten den text des buches einmal gründlich um, statt die berichtigungen in anmerkungen, oft in anmerkungen zu anmerkungen zu bringen!

7) Grimm gramm. I, 222 und 506. Das mittelenglische gibt Grimm leider nur nach Tristrem, Alisaundre und Chaucer, es liess sich natiurlich 
schrieben, sowol diejenigen, welche sich des normannisch-französischen, als die, welche sich des (neu-) angelsächsischen bedienten. ${ }^{9}$ )

Latham hat zuerst darauf aufmerksam gemasht, wie wenig doch eigentlich das normannisch-französische auf die angelsächsische sprache eingewirkt hat. ${ }^{10}$ ) Er führt aus, wie das angelsächsische nach natürlichem gesetze sinken muste, auch ohne die normannische eroberung und tritt so gegen die auf, welche behaupten wollen, das abschleifen der endungen, das abschwächen der vollen vocale und ähnliches sei durch die fremden gekommen. Madden in seiner trefflichen einleitung zu Lazamon folgt Latham und stellt seit der normannischen eroberung folgende perioden auf: ${ }^{11}$ )

$$
\begin{aligned}
& 1100 \text { - } 1230 \text { Semi-Saxon. } \\
& 1230 \text { - } 1330 \text { Early English. } \\
& 1330 \text { - } 1500 \text { Middle English. } \\
& 1500 \text { - } 1600 \text { Later English }
\end{aligned}
$$

Im allgemeinen ist diese art der einteilung jetzt uberall angenommen. Zweifel herscht uiber die letzte periode, die meist schon zum neuenglisch gerechnet wird. Ebenso kann man über 1100 als beginn des Semi-Saxon streiten, allein diese aufstellungen sind ja alle nur ungefähr und uberall gibt es hier ubergänge, die man zur früheren oder späteren periode zählen kann.

Koch hat ebenfalls diese einteilung, welche Madden machte, angenommen, doch weist er mit recht den eigentümlich gewählten namen halbsächsisch (den Mätzner beibehalten hat ${ }^{12}$ ) zurtick. ${ }^{13}$ )

nach diesen quellen, welche zeitlich und örtlich so sehr auseinander liegen, keine einheitliche darstellung der sprache erzielen.

8) Vgl. Wright Biographia Britannica Literaria. I. the Anglo-Saxon period. II. the Anglo-Norman period.

9) Ebend. II. bd. Hier findet sich neben Wace, Turold (d. h. also dem dichter des Rolandliedes), Philipp de Thaun, Wilhelm von Malmesbury, Gervasius von Tilbury, als endlich Lazamon, Orm uud Nicholas de Guildford (als dichter der, eule und nachtigal “ s. unten).

$\left.{ }^{10}\right)$ Latham, the English Language. London 1841. pag. 61. ff.

11) Madden in der ausgabe des Lazamon (vgl. unten) pag. VI.

12) Mätzner in seiner englischen grammatik p. 6.

13) Koch in seiner historischen grammatik der englischen sprache pag. 8. 
Nach wie vor ist die flexion der verba und nomina durchaus angelsächsisch, die romanischen bestandteile des wortschatzes sind vollkommen germanisiert, Semi-Saxon aber könnte leicht vermuten lassen, die sprache von 1100-1230 sei eigentlich kein angelsächsisch mehr. Koch führt die bezeichnung neu-angelsächsisch ein und ihm ist aus obigen grüden entschieden beizustimmen.

In der nun folgenden zeit wird der verlust der Normandie unter Johann für die entwicklung der englischen sprache wichtig, fast noch wichtiger aber das von Heinrich III. und Ludwig IX. gegebne gesetz, dass kein edler zugleich in England und in der Normandie land besitzen dürfe. Hierdurch hörte der zuzug normannisch sprechender barone auf. Die folgen dieser ereignisse zeigten sich natürlich nicht sofort. Zum glücke für das germanische element brach bald grosse unzufriedenheit unter den normannischen grossen mit der regierung Heinrichs aus. Es musten sich nun die Normannen, um mit erfolg den kampf mit dem könige aufnehmen zu können, auf das angelsächsisch redende volk stutzen. Der hass zwischen Normannen und Angelsachsen wurde aufgehoben und das germanische und romanische sprachelement im englischen ${ }^{14}$ ) ausgeglichen.

14) Über den namen "englisch " sei hier eine bemerkung vergönnt. Mätzner sagt a. a. o. pag. 6: „Der bildung der englischen sprache geht eine tibergangsepoche, die des halbsächsischen voran - die sprache nennt sich allerdings schon englisch." Zum belege wird dann angeführt: Orm Ded. v. 322.

Jcc patt tis Ennglissh hafe sett

Ennglisshe menn to lare.

Es liessen sich aus Orm noch viele stellen dafür anfuhren Ded. v. 109, 113, 147, 157, 306, 317, 331 u. a. Bei Laz. heisst es v. 31:

He nom pa Englisca boc, pa makede Seint Beda.

Weiter findet sich in Juliane, dass der verfasser sein werk „of Latin iturnd to Englische leode“ nennt (vgl. unten). Ebenso S. Marh. pag. 23: ipe moneð pet on ure ledene is ald englisch efterlið inempnet. Doch schon altags. haben wir belege: in der übersetzung des ev. Nic. (Thwaites gab es heraus) wird z. b. cap. XXI gesagt: Tollite portas prin-

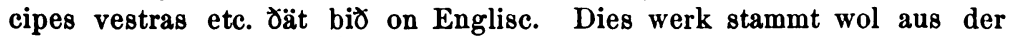
1. hälfte des 11. jh. Älfric in seiner übersetzung der Genesis (Grein, ags. prosa I, 22): pu bæde me, pät ic sceolde âvendan of Lêdene on Englisc pâ bôc Genesis. Auch sonst nennt er seine sprache Englisc p. 6, 7, 15 ebend. Doch schon früher uiberträgt Älfred in Beda stets 
Die haupteinwirkungen des romanischen auf das angelsiächsische sind:

In der declination des plurals bewirkt das normanische, dass man beim mascul. die starke endung auf es beibehielt, die ursprïnglich sw. masc. aber mit dieser endung versah, ebenso den femininen und neutren, mit wenigen ansnahmen, diese pluralendung gab. Im sing. blieb hingegen das deutsche genitiv-s und drang auch in das femininum vor. Die beugung der adjective ist noch heutigen tages deutsch. Nur ist vielleicht das verstummen des flexivischen $e$ romanischem einflusse zuzuschreiben.

Durchaus deutsch blieben ferner zeitwort, furwort und zahlwort. Ebenso der grōste teil der adverbien und praepositionen.

Der haupteinfluss des französischen zeigt sich in den lauten, vor allem in den gutturalen, und besonders in dem wortvorrate.

Die zeit nun, wo sich das germanische mit dem romanischen auszugleichen sucht, also die periode von c. 1240-1517; teilt man gewöhnlich in zwei abteilungen, in die altenglische (-1330 nach Madden) und in die mittelenglische (-1500). Der unterschied zwischen beiden ist nicht so leicht festzustellen. Doch setzen wir 1350, nicht 1330, als anfang der mittelenglischen zeit, so zeigt sich doch eine grosse verschiedenheit. Die dichter der altenglischen zeit schreiben ihre heimischen dialecte, Robert von Glocester im sudwestdialect, Robert Mannyng im Lincolndialect, Wilhelm von Shoreham, Dan Michel im Kentdialect, Richard Rolle de Hampole und Lorenz Minot in nordenglischer sprache.

Hingegen in der mittelenglischen periode suchen die schriftsteller, wenigstens die bedeutendsten, durch aufgeben eines teiles ihrer dialectischen eigentumlichkoiten sich allen Engländern verständlich zu machen. Allerdings ist auch nicht aus dem auge zu lassen, dass Langley, ${ }^{15}$ ) der verfasser der visions concerning Piers the Plowman, in Shrop, Chaucer, Gower, Lidgate, in Kent lebten, Wycliffe hingegen, obgleich in Yorkshire geboren, Trevisa,

Saxonica lingua: on oder in Englisc, ebenso praef. zur ubersetzung des Beda.

15) Dass der verfasser dieser schrift Langley, nicht Langland hiess, bat Pearson bewiesen. North British Review 1871 p. 241-245 und Early Engl. Text Soc. 7. Report of the Committee (February 1871) p. 8. 
der ubersetzer des Higdenschen werkes, der aus Cornwales stammt, wenigstens sich längere zeit in London und Oxford aufhielten und so alle mehr oder weniger in der sprache von Kent und von Mittelengland schrieben.

Die sprache ist allerdings auch in der mittelenglischen periode noch weit entfernt $\mathrm{zu}$ einer vollkommnen einheit gelangt zu sein und erst den geisteshelden des 16. und 17. jahrhunderts gelang es, hier durchgreifend einzuschreiten und so die zeit, welche wir die neuenglische nennen, heranzufuhren.

Koch und Mätzner haben in ihren grammatiken das neuangelsächsische nach seinem bedeutendsten poetischen denkmale, dem Brut des Lazamon, dargestellt. Ihm gegenüber wird dann, als vertreter des nordhumbrischen, die homiliensammlung Orms gesetzt. ${ }^{16}$ )

Doch seit erscheinen dieser grammatiken haben englische gelehrte eifrig durch herausgabe von texten und auch von grammatischen arbeiten die kenntnis des neu-angelsächsischen gefördert. Es sind hier drei namen zu nennen: James Morton, Oswald Cockayne und Richard Morris. Durch ihre veröffentlichungen hat sich die kenntnis des nags. sehr erweitert und die angaben, welche Koch macht, bedürfen öfters sehr der ergänzung. ${ }^{17}$ )

Selbstverständlich soll daraus Koch durchaus kein vorwurf gemacht werden. Er hat mit den mitteln, welche ihm geboten

16) hg. sind die homilien: The Ormulum, now first ed. from the Bodleian ms. by Robert Meadows White. 2 bde. Oxford 1852. Schon Hickes (oder besser Wanley) hat nachricht darüber gegeben.

17) Um hier nur eins anzuftihren, ist tiber den gebrauch des duals der pronomina im nags., also zu Koch pag. 467 \& 161, viel nachzutragen. Orm verbindet den dual des pron. hänfig mit twa oder ba z. b. Ded. 6: witt ba, 8655 witt bape, zunnc bape 4495 , zitt bape 6206, 6247 etc. Doch ohne weitere zufügung steht der dual der pron. pers. Ded. 73, Hom. 201, $202,4498,12362,12363,13012,13014,13020,6242,8663$ u. a. Einzelne dualformen finden sich noch während der ganzen nags. zeit z. b. H. Meidenh. inc p. 11; inker 31. 0. a. N. unker 552, 151, 993, 1689, 1780, 1782, 1783. hunke (= zunnc bei Orm) 1733. Diese letzte form zeigt sowol Calig. A. IX als ms. Jes. Coll. Arch. I, 29. Stratmann ändert ganz falsch und willkürlich in unk, er miskennt form und sinn. Mätzner hat sie richtig erklärt, sprachpr. I, 1 pag. 48. Unk ist ganz sinnlos, denn den vers 1733 spricht der zaunkönig (pe wrenne) und unter: hunke ist natiirlich eule und nachtigal zu verstehen. 
waren, so bedeutendes geleistet, dass jeder, der fernerhin ther englische sprache arbeiten wird, sich an Koch anschliessen muss.

Lazannons chronik ist nach wie vor das bedeutendste werk aus der nags. zeit geblieben, doch ihm an die seite ist ein prosaisches werk getreten, be Ancren Riwle, das sprachlich nicht weniger interessant ist, als Lazamon. Sachlich allerdings haben viele der anzuführenden schriften wenig bedeutung.

Es scheint, dass nach der eroberung Englands durch die Normannen die angelsächsische nation längere zeit keinen schriftsteller hervorgebracht hat. Will man nicht die fortsetzung der Sachsenchronik oder vielleicht einige homilien hierher setzen, so finden wir, abgesehen von urkunden, in den ersten siebzig jahren des 12. jahrhunderts keinen rest der ags. sprache. Am hofe bluhte französische poesie, die angelsächsischen grossen, welche vorher die dichtung begtinstigt hatten, waren unterdrückt oder irrten als verbannte in Nordengland umher, selbst in den klöstern wurde das angelsächsische element in jeder weise unterdritckt, das volk aber mag sich, wie dies immer bei unterdrückten völkern geht, lieber am ruhme der vorzeit erfreut und den alten heldenliedern gelauscht, als neues gedichtet haben.

Erst am ausgange des 12. jahrhunderts lassen sich wieder literarische produkte feststellen.

Vor Lazamon ist vielleicht noch zu setzen:

I. Seinte Marherete pe meiden ant martyr ${ }^{18}$ )

Das leben der Margarete zu bearbeiten scheint im M. A. sehr beliebt gewesen zu scin. Ausser den verschiednen deutschen und französischen übertragungen besitzen wir auch eine alt-angelsächsische. ${ }^{19}$ ) Das nun hier anzuftuhrende leben der Marg. ist in alliteration geschrieben und darf noch in das zwölfte jahrhundert gesetzt werden; Cockayne aber nimmt sicher eine zu fruhe zeit an. ${ }^{20}$ ) Erhalten ist es uns in zwei hss. Die eine aus dem

18) Hg. von Oswald Cockayne: Seinte Marherete the meiden ant martyr. London 1866. Early Engl. Text Society No. 13.

$\left.{ }^{19}\right) \mathrm{Hg}$. von O. Cockayne in Narratiunculae Anglice conscriptae.

20) Cockayne sagt in der ausgabe der Marherete: pag. VI: Sir F. Madden (Lazamon III p. 350 - dies soll heissen: 359) has stated that ;he piece (viz. the life of Margaret) was probably composed about 1200 ᄂ. d., and as it seems in some respects a few years older than the prin- 
anfange des 13. jahrhundert ist Oxf. Bodl. No. 34., die andre Brit. Mus. Reg. Ms. 17 A 27 (fol. 37-56 enthalten das leben). Diese hs. ist etwa 1230 entstanden. ${ }^{21}$ )

II. The proverbs of King Alfred. ${ }^{22}$ )

Gleichzeitig oder etwas früher als Lazamon ist eine sammlung von denksprüchen und sprüchwörtern, welche sämmtlich

ted earlier text of Layamon, it will be as well to acquiesce in that opinion. Sir Frederic is well able to maintain any opinion he forms, but if compared with the text of the last entries in the Chronicle written soon after 1154 and before 1177, the language of St. Marherete might be put thirty or forty years earlier. - Cockayne nimmt also etwa 1165 an. Besser ist es wol mit Madden das ende des 12. jh. für die entstehung anzusetzen.

21) Vgl. Morris: Old English homilies and homiletic treatises of the $12^{\text {th. }}$ and $13^{\text {th. }}$ centuries ed. by Rich. Morris. First series. part. I Earl. Engl. T. Soc. No. 29. London 1869 pag. IX.

22) Zwei hss. sind uns davon erhalten: Ms. Trin. Coll. Camb. B. 14, 30 aus dem anfonge des 13. jh. und Ms. Coll. Jes. Oxf. I, 29, abgedruckt in den Reliquiae Antiquae ed. by Wright and Halliwell I, $171 \mathrm{ff}$. Eine dritte hs. Galba A, XIX ging bei dem brande der Cottoniana verloren. Wanley bei Hickes gibt die ersten zeilen I, pag. 231. Diese hs. gehörte zu einer gruppe mit dem Jes. Coll. ms. Man vergleiche

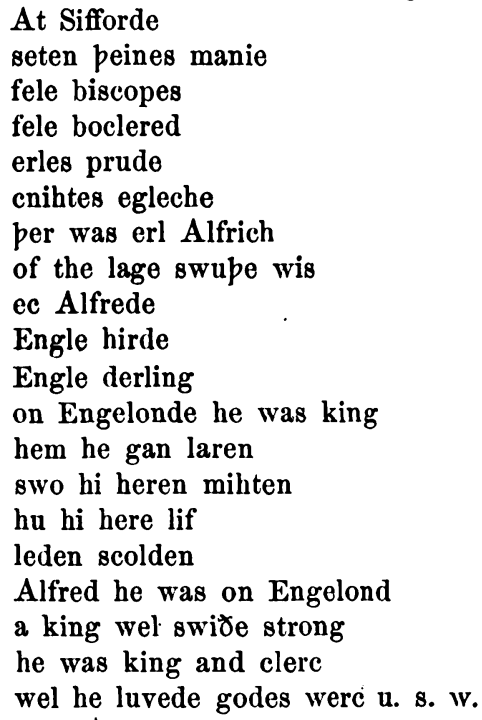

Der schluss der hs. ist leider nicht angegeben. 
beginnen: „pus quep Alfred.“ und am anfange wird ausdrucklich der könig Alfred genannt.

In welchem zusammenhange diese sprichwörter mit Lazamon stchen, ist noch zu untersuchen.

So finden sich (p. 185.) die zeilen:

And hweder so pu hwendes,

sei pu aten ende:

wrpe pat iwurpe

iwurpe Godes wille

und Lazamon schliesst seinen Brut

$$
\begin{aligned}
& \text { iwurde pet iwurde } \\
& \text { iwurde Godes wille. }{ }^{23} \text { ) }
\end{aligned}
$$

Ebenso beruft sich im gedichte ${ }_{n}$ eule und nachtigal ${ }^{\text {" sowol }}$ die letztere als die eule häufig auf Alfreds sprïche. ${ }^{24}$ ) Allerdings können darunter auch alt-angelsächsische gemeint sein.

\section{Lazamons Brut. ${ }^{25}$ )}

Erhalten ist uns das werk in zwei bearbeitungen, die ältere bearbeitung im Ms. Brit. Mus. Cott. Caligula A IX, hs. aus dem anfange des 13. jahrhunderts (nach Madden). fol. 1-192 enthalten die dichtung. Die andre bearbeitung ist aus dem ende des 13. jahrhunderts und bedeutend verkürzt. Sie ist Cott. Otho C XIII. Durch den brand, oktober 1731, hat sie bedeutend gelitten.

Wir wissen über Lazamons leben nur das, was er uns selbst dariber angibt v. $1-8$.

$$
\begin{aligned}
& \text { An preost wes on leoden } \\
& \text { Lazamon wes ihoten, } \\
& \text { he wes Leovenares sone: }
\end{aligned}
$$

23) Vyl. Laz. ed. Madden III, 297. Oder bezieht sich die anu. zu v. 32:35: .. addled by a later hand in the margin" auf den ganzen schluss nnd wären dann die schlusszeilen später zugefïgt? Dies ist unglaublich nach lem, wie Wanley bei Hickes III, 228 den schluss gibt:

And pas (leode) pat nevere zeodjen mære kinges neoren here da zet ne com pet ilke dæi beo heonne voro alse hit mei. Jwurde godes wille. Amen. - Wanley hat hier nur ,leode" eingeklammert, doch gibt er llerdings gerade unsre stelle anders.

21) Vgl. (. a. N. v. 235, 294, 299, 349 u. a. a. o.

2i) Lazamons Brut or chronicle of Britain, a poetical Semi-Saxon garaphrase of the Brut of Wace. 3 bde. Iondon 1847. - Hier sind beide iss. abgedruckt. 
liðe him beo drihten.

he wonede at Ernleze

at æðelen are chirechen

uppen Sevarne stape,

sel par him puhte.

on fest Radestone,

per he bock radde.

Madden hat gezeigt, dass dieses Ernley dasjenige sei, welches in Worcester, nicht das, welches in Stafford, liegt. ${ }^{26}$ ) Laz. führt Beda, Albinus und Augustinus, endlich Wace als quellen an. Letzteren hat er zu grunde gelegt, Beda hat er nur einmal nachweislich benutzt, ${ }^{27}$ ) wie Madden schon ausgefuhrt hat, die anderen gar nicht. Doch Wace ist nichts weniger, als wörtlich ubbersetzt, Lazamon hat sehr erweitert, vor allem hat er viele ortssagen und legenden aufgenommen und besonders die Artussage mit vielen zügen bereichert, die sich auch nicht bei Gotfried von Monmouth finden. ${ }^{28}$ )

Über die zeit der abfassung hat Madden scharfsinnig aus den durftigen angaben festgestellt, dass in den ersten jahren des 13. jahrhunderts das werk noch nicht vollendet war, dass es aber vor 1205 abgeschlossen wurde. Da aber ein so grosses gedicht jahrelangen fleisses bedurfte, gewinnen wir das ende

26) Madden, preface $X$.

27) Laz. sagt v. $31 \mathrm{ff}$. von sich:

He nom pa Englisca boc, pa makede seint Beda.

an oper he nom on Latin, pe makede seinte Albin and pe feire Austin, pe fulluht broute hider in. boc he nom pe pridde, leide per amidden, pa makede a Frenchis clerc, Wace wes ihoten.

Über Austin u. Albin vgl. Madden XII. Dass Wace seine hauptquelle war, deutet Lazamon selbst durch das "amidden“ an.

${ }^{28}$ ) Es ist dies ein beweis, dass noch andre quellen fïr die geschichte Arturs in England existierten, als Gotfried von Monmouth. Sonst wïre auch schwer das plötzliche emportauchen der vielen Artussagen im 12.jh. zu erklïren. 
des 12. und den anfang des 13. jahrhunderts für die zeit der abfassung. ${ }^{29}$ )

Die alliteration waltet vor, doch finden sich auch assonanzen und selbst reime.

IV. Etwas später als das leben der heiligen Margarethe sind zwei andre heiligenleben anzusetzen, die wol am anfange des 13. jahrhunderts abgefasst wurden.

\section{Seinte Katherine. ${ }^{30}$ )}

Es ist uns in drei hss. erhalten. 1. Bodl. No. 34. (fruher NE. 3, XI.) aus der zeit Johanns oder Heinrichs III. ${ }^{31}$ ) beginnt: Constantin ant Maxence were on a time as $\mathrm{i}$ keiseres stude hehest i Rome, ah Constantin ferde purh pe burhmenne into Franclonde ant wunede sumne while pear for be burhes neode ant Maxence steorede pe refschipe i Rome. Weox umbe hwile wreaððe ham bitweonne ant comen to fehte. Wes Maxence overcumen and fleah into Alexandre. . . Schluss: pus wende pe eadie meiden Katerine icrunet to Criste from eoroliche pinen $i$ Novembre moneð pe fif and twentesðe dei and fridei on outte under $\mathrm{i}$ pe dei $\mathrm{i}$ pe tune $\mathrm{p}$ hire deore leofmon Ihs ure laverd leafd lif o rode for hire and for us alle. Beo he a se halend iheret and iheiet in alre worlde worlt a on ecnesse. Amen.2. Ms. Reg. 17 A XXVII fol. 11-37. (c. 1230 entstanden.) hiernach hat Morton seinen text gegeben. ${ }^{32}$ ) 3. Eine dritte hs. 'Titus D XVIII., die auch sonst sehr wichtig für nags. ist, schliesst sich, algesehen von lautlichen abweichungen, dem Bodl. ms. an. ${ }^{33}$ )

$\left.{ }^{29}\right)$ Vgl. Madden pag. XVII-XXI.

${ }^{30}$ ) Zweimal ist das leben der Katharina herausgegeben worden: James Morton veröffentlichte es London 1841 für den Abbotsford Club (nur fiir mitglieder zu bekommen!). Dann: An historial inquiry touching st. Catharine of Alexandria, to which is added a Semi-Saxon legend by ('harles Hardwick. Cambridge 1549 fiir die Antiquarian Society. No. XV.

31) Wanley bei Hickes III p. 79.

32) Vgl. Cockayne S. Marh. pag. VI.

$\left.{ }^{33}\right)$ Hickes III, 247. Der inhalt der hs. ist:

1. Liber Alphabetarius.

2. Ancren Riwle.

3. Homilie: Si sciret paterfamilias.

4. Audi filia et vide et inclina etc. (vgl. unten. Es ist dies also die homilie Hali meidenhad).

5. pe wohunge of ure laverd.

6. passio St. Catherinae. 
V. Seinte Juliene. ${ }^{34}$ )

Bodl. ms. No. 34. enthält auch dieses heiligenleben. fol. 36b-56.a Es beginnt:

I pe feaderes ant $\mathrm{i}$ pe sunes and $\mathrm{i}$ pe hali gastes nome her beginneð pe liflade ant te passiun of Seinte Juliene. In ure laverdes lufe pe feader is of frumscheft ant $i$ pe deore wurtmunt of his deorewurde sune ant $i$ pe heiunge of pe hali gast pe of ham ba glideð an Godd unazin euch godes ful. Alle leawede men pe understonden ne mahen Latines ledene liðe $\delta$ ant luste $\circlearrowright$ pe liflade of a maiden pæt is of Latin iturnd to Englische leode. - Es schliesst:

Hwen drihtin o domes dei windwer his hweate

And weorpd pæet dusti chef to hellene heate,

He mote beon a corn i godes guldene edene,

pe turde pis of Latin to Englische ledene

Ant he pæt her least onwrat swa as he cuð.

Eine andre hs. soll sich in der Cottoniana finden, welche Brock mit Cockayne herausgeben wird. ${ }^{36}$ ) Auch im schon genannten ms. Reg. 17 ist fol. $56-70^{b}$ dies heiligenleben $\mathrm{zu}$ finden. ${ }^{37}$ )

VI. pe ule and pe nihtegale. ${ }^{38}$ )

Zwei hss. sind uns davon bekannt. Eine Brit. Ms. Cott Calig. A IX. ${ }^{39}$ ), die andre Oxf. Jes. Coll. Arch. I, 29.

Wann dieses gedicht entstanden sei, ist sehr verschieden beantwortet worden. Stevenson setzte es an das ende des 12 .

अ) Hiervon gibt es noch keine ausgabe. Doch soll demnächst eine von Cockayne erscheinen, vgl. Eighth report of the Earl. Eng. T. S. 1872 pag. 5.

35) $\mathrm{Vgl}$. Hickes III, p. 79.

${ }^{36}$ ) Vgl. den oben ang. report of the E. E. T. S. pag. 5.

${ }^{37}$ ) Cock. S. Marh. pag. VI.

${ }^{38}$ ) Zwei drucke haben wir nach; der Cott. hs. The Owl and the Nightingale. Ed. by Jos. Stevenson. London 1838. $4^{0}$. Printed f. th. Roxburghe Club (einige lesarten des Jes. Coll. finden sich hier). The o. a. t. N. on early poem attributed to Nicholas de Guildford ed. by 'Thom. Wright. London 1843. $8^{\circ}$. Pr. f. t. Percy Society. Beide hss. hat abgedruckt mit wenig verbesserungen und manchen willkürlichkeiten: J. H. Stratman : an old English poem of the Owl and the Nightingale. Krafeld 1868.

39) Schon Wanley gibt nachricht dariber III, 229. 
jahrhunderts. Dies ist den reimen nach zu fruh, denn die reime sind gröstenteils recht rein, wenn auch öfters sich noch assonanz einschleicht. ${ }^{40}$ ) Wright glaubt, dass es unter Johann ohne land entstanden sei. ${ }^{41}$ ) Madden nimmt an, es sei erst unter Eduard I. gedichtet worden. ${ }^{42}$ ) Dies wäre jedenfalls zu spät und gradezu unhaltbar, wenn es sich erweist, dass das Cott. ms. aus der 1. hälfte des 13. jahrhunderts stammt. Marsh lässt es ziemlich unbestimmt, wann er das werk abgefasst glaubt. Er gibt nur das 13. jahrhundert an, stellt es aber zwischen King Alisaundre und Horn child. ${ }^{43}$ )

Einige gelehrte schreiben das gedicht Niclas de Guildford zu. Hickes hat diesen fehler nicht begangen, ${ }^{44}$ ) jedoch Warton ${ }^{45}$ ) teilt es auf ganz falsche schltisse hin einem Johannes de Guldevorde zu. Madden wies schon in der ausgabe W's von 1840 nach, wie unberechtigt dies sei. Wright meint, Nicolas de Guildford sei der verfasser. ${ }^{46}$ ) Nicholas wird aber stets in

10) Es ist daher auch jedenfalls von Stratmann unberechtigt, dass er öfters gegen die hss. ändert, um einen reim herzustellen. Wo reime vorkommen, wie cunde: schende 273 ; fuzele: uvele 277 ; gidie: zeonie 291 ; worse: mersche 303 u. ähnl. braucht man wahrlich nicht dem reime zu lieb zu ändern.

11) V. 1091 (ich citiere nach Stratmann) steht:

pat underwat pe King Henri

Jesus his soule do merci.

Dies bezieht Madden auf Heinrich III. und es wäre nach dessen tode tnter Eduard I. abgefasst. Stevenson und Wright sehen in diesem Heinsich Heinrich II. ( +1189$)$, Wright setzt das gedicht aber erst unter Johann an, vgl. Wright Biographia Britannica literaria. Anglo-Norman period. London 1846 . pag. 438.

42) Madden in den anm. zu Warton pag. 26.

13) Marsh, the origin and history of the English language and of the tarly literature it embodies. New-York 1862. p. 205-211. Da Alisaundre Ind Horn erst ende des 13. jh. gedichtet wurden, folgt er wahrscheinlich Iadden.

41) Wanley sagt p. $229 \mathrm{III}$ : iiber unser gedicht: Poema Normannosaxonicum de contentione facta inter noctuam et philomelam pro suavisrimae cantionis gloria, quam demum submiserunt arbitrio magistri Yicolai de Guldeford.

45) Vgl. W. hist. of poetry I, $25 \mathrm{ff}$.

46) Wright a. a. o. sagt: The name of Nicholas de Guildford occurs is a poem ... in a way which would lead any one acquainted with the 
einer weise genannt, dass er unmöglich der verfasser sein kann, oder er müste einer der eitelsten menschen gewesen sein. ${ }^{47}$ )

Ausserdem sagt der dichter am anfange:

Ich was in one sumere dale:

In one swipe dizele hale

Iherde ich holde grete tale

An ule and one nihtegale

Von Nicholas wird stets als von einem abwesenden gesprochen, der zu Portesham in Dorset wohnt und zum schlusse heisst es:

1789. Mid pisse worde forp hi ferden

Al bute here and bute ferde

To Portesham pat heo bicome

Ac hu heo spedde of heore dome

$\mathrm{Ne}$ can ich eu na more telle

Her nis na more of pisse spelle.

Warum liesse der dichter, wenn er mit Nicholas eine person wäre, die vögel wegziehen, statt aus seinem verstecke hervorzutreten oder warum könnte er uns nicht den urteilsspruch berichten?

Jedenfalls ist das richtige einen unbekannten dichter anzunehmen, der dieses werk Nicolaus von Guildford $\mathrm{zu}$ ehren schrieb, in bezug auf die zeit der entstehung hat Wright wol das richtige getroffen.

VII. Es sei hier noch ein andres gedicht angeschlossen, welches in alliteration geschrieben ist. Doch uns ist nur ein bruchstluck davon erhalten. Conybeare nennt es:

Poem on Death, qder the Grave, wie es Thorpe nennt. ${ }^{48}$ ) Dies gedicht ist auf den rand eines homiliencodex (Nags. homilien) geschrieben. ${ }^{49}$ ) Bodl. NE. F. 4, 12.

manner in which writers of the middle ages name themselves, to believe him to be the author. In den Chronological series of the literary characters finden sich (pag. i481) zwischen 1174-1228: Orm, Nicholas de Guildford, Lazamon.

เ7) Man vergleiche die stellen, wo von Nicholas die rede ist, จ. $191,1755$.

48) Conybeare Illustrations of Anglo-Saxon poetry. London 1826. pag. 270 ff. - Thorpe, Analecta Anglo-Saxonica. London 1834.

49) Abgedr. findet es sich auch bei $M$. Rieger, alt- und angelsächsisches lesebuch. Giessen 1861. p. 124. 
Es beginnt: pe wes bold gebyld, er pu iboren were, pe wes molde imynt er pu of moder come.

Conybeare lässt die letzten drei langzeilen hinweg. Sie lauten nach Thorpe:

for sone bið pin hæfet faxes bireved

al bið pes faxes feirnes forsceden, næle hit nan mit fingres feing stracien. ...

VIII. pe Ancren riwle. ${ }^{50}$ )

Dieses bedeutendste prosadenkmal der nags. literatur 'ist uns in vier mss. erhalten, welche zwei gruppen bilden.

Zur ersten gruppe gehören die drei Cottoniana.

Nero A XIV aus dem XIII. jh. pg. 8. 131 bl. 51). Diese hs. hat Morton seiner ausgabe zu grunde gelegt. - Titus D XVIII. pg. $8.147 \mathrm{bl} .{ }^{52}$ ) Am anfange fehlen einige blätter. Ms schliesst: Ase ofte as ze haven red oht 0 pis boc gretes ure lavedi wir an ave for him (viz. pet maked peos riwle) and swanc her abuten, ${ }^{53}$ ).- Cleopatra CVI, 40201 bl. ${ }^{54}$ ) Beginnt: Recti diligunt te. Laurd seið godes spuse to hire deorewurðe spuse . . . Schluss: As ofte as ze habbed capet (oder cawet soll wol owet heissen) peron greteð pe lafdi wið an Ave for him, pe swong her abuten. Inoh meðful ic am, pe bidde pe lutel.)

Die zweite gruppe ist nur durch eine hs. vertreten:

Cambridge, Corp. Christi coll. No. 402, 40. pg. ${ }^{55}$ ) Diese hs. weicht sprachlich bedeutender ab und es ist daher schade, dass Morton in seiner fleissigen ausgabe sie nicht mehr beachtet hat. Sie trügt einen etwas mehr westmitteländischen stempel

50) The Ancren riwle, a treatise on the rules an duties of monastic life ed. by James Morton. London 1852. pr. f. the Camden Society.

51) Vgl. Hickes III, p. 228, Planta, Catal. der Cotton. London 1802. pag. 205. Ms. enthält:

Ancren Riwle, Marienlied (Christes milde moder seinte marie, mines lives leome, mi leove lefdi), $120^{\mathrm{b}}$; Orationes ad Christum et Mariam (englisch) $123^{\text {b }}$; symbolum apostolicum 130 bis schluss.

52) Hickes III, pag. 247, vgl. anm. 33 .

53) Vgl. damit pag. 430 bei Morton.

54) Hickes UI, 248. Planta pag. 581; bl. 3-198 enthält Ancren riwle. Es folgt hymnus in solemnitate S. Ethelredae.

55) Hickes III, 149. Das werk nennt sich Ancren wisse. 
(damit stimmt auch die notiz die sie trägt, ${ }^{56}$ ) überein), während die andern slidlicher entstanden sind.

Endlich besitzen wir noch einen druck Bibl. Bodl. Laud. D. 85. ${ }^{57}$ ), welcher gebete aus der Ancren riwle enthält. Sie gehören zur zweiten gruppe, vielleicht sind sie geradezu aus obigem Cambridge codex entnommen.

1. Ealmihtig god fæder suna and hælig gast as ze beoð an god ealra etc. (= pag. 26 bei Morton).

2. La ihu, pin are for myne synnas ahongen 0 rode. (= pag. 26).

3. For pa seovengyftas pæs halzan gastes. (= pag. 28).

4. For pa tyn heastas pe ic gebroccen hæbbe. (= pag. 28).

5. For pa wurdegunge ihu crist of pine tweolfen apostolas (= pag. 28).

6. For ealle pe sawlen pe beoð forð faren i pe bileave of pa feower godspelles. (= pag. 30).

Leicht überzeugt man sich aus diesen anfängen, dass hier eine ältere sprache vorliegt, als in den Cotton mss. Um so verdienstroller wäre es, den text der Cambr. hs., der hier wahrscheinlich zu grunte liegt, herauszugeben.

Viel ist darüber gestritten worden, ob unser werk ursprünglich nags. abgefasst wurde, oder ob es nur eine ubersetzung aus dem lateinischen. Thomas Smith ${ }^{58}$ ) hält den nags. text nur für eine tibersetzung aus dem lateinischen und dieses lateinische original will er gefunden haben in der hs. Cotton. Vitellius EVII. Zu dieser ansicht brachte ihn wol nur die aufschrift des codex. ${ }^{59}$ ) H. Wanley ${ }^{60}$ ) bekräftigt diese behauptung auf grund einer vergleichung, welche er zwischen Vit.

56) Sie lautet: Liber ecclesie sancti Jacobi de Wigmore quem Johannes Purcel dedit eidem ecclesie ad instantiam fratris Walteri de Lodelle senioris tunc percentoris etc. Wigmore liegt in der Grafschaft Hereforde.

$\left.{ }^{57}\right)$ Hickes III, $100 \mathrm{f}$. Das buch hat die notiz: Imprimatur: Henry Butts, Procanc. Cantabrig. 3. dez. 1630. Es enthält: the Anglo-Saxon psalter, dann Certaine prayers of the Saxon times taken out of the Nunes rules of St. James Order in Bennet College library.

$\left.{ }^{58}\right)$ Smith catalogus bibliothecae Cottonianae. Londini 1696.

59) Regulae vitae Anachoretarum utriusque sexus scriptae per Simonem de Gandavo, Episcopum Sarum in usum suarum sororum.

(n) Hickes bd. III, p. 228. 
EVII. und den Cotton ms. angestellt haben will. Ihm folgt Planta. ${ }^{61}$ )

Was zuerst den angeblichen verfasser des originals, Simon von Gent, bischof von Salisbury betrifft, so lässt sich nur einer nachweisen, welcher 1284 archidiacon zu Oxford, 1297 bischof von Salisbury wurde und 1315 starb. Der vater desselben kam aus Gent, daher wol der name. ${ }^{62}$ )

Die sprache der Ancren riwle aber ist nicht altenglisch, sondern neuangelsächsisch. Simon von Gent kann also nicht der verfasser des originals sein.

Allerdings könnte nun, wenn auch Simon nicht den lateinischen text verfasst hat, doch das original lateinisch gewesen sein. Leider ist der Cod. Vit. EVII. bei dem brande, oktober 1731, zu grunde gegangen. Doch eine andre hs., welche Smith und Wanley kennen, ist uns erhalten: ms. Magdalene College zu Oxford. ${ }^{63}$ ) Sie führt die aufschrift:

Hic incipit prohemium venerabilis patris magistri Simonis de Gandavo, Episcopi Sarum, in librum de vita solitaria, quem scripsit sororibus suis anachoretis apud Tarente.

Es ist ein verdienst Mortons mit schlagenden gründen nachgewiesen $\mathrm{zu}$ haben, dass diese lateinische hs. nur eine ubersetzung und zwar eine recht schlechte des nags. textes ist, die von groben fehlern und misverständnissen wimmelt. Ausserdem ist das erste buch stark gekürzt, das achte fehlt gänzlich. ${ }^{64}$ )

Schliesslich könnte man noch ein französisches original annehmen und wirklich gab es auch das werk in franzōsischer sprache. ${ }^{65}$ ) Mätzner fulhrt gegen das franz. original die stelle p. 44 an: $\left.{ }^{66}\right)$

Al pet ze ever siggeð of swuch oðer bonen, ase of Paternostres and of $\Lambda v e z$, on ower owene wise, psalmes and ureiguns: al ich am wel ipaied everichon sigge pet hire best bered in heorte: verslunge of hire sautere: redinge of Englichs oðer of Freinchs, holi medidaciouns.

${ }^{61)} \mathrm{Vgl}$. a. a. o. p. 205.

62) Vgl. Morton preface XIV.

${ }^{63}$ ) Ebend. VII.

iA) Ebend. pag. VIII ff.

(:) Vitellius F VII bei Smith a. a. 0. Sie ist 1731 verbrannt.

u) Mätzner, altenglische sprachproben I, 2. abt. Berlin 1869. pag. 6. 
Mätzner meint, nonnen, welche sowol englisch als französisch verstanden hätten, wäre es nicht nötig gewesen, etwas aus dem französischen zu ubertragen. Doch, wenn das werk auch zunächst an drei schwestern gerichtet ist, welche wol beide sprachen verstanden, geht doch aus andern stellen hervor, dass der verfasser auch auf verbreitung seiner schrift in grösseren kreisen rechnete. ${ }^{67}$ ) Und ob alle drei schwestern französisch lesen kounten, ist nirgends gesagt. Jedenfalls hätte man immer in hinblick auf grösseren zuhörerkreis das werk in das englische ubertragen können.

Allein der ton des ganzen werkes ist durchaus englisch. Die romanischen wörter sind allerdings zahlreich, allein bei einer geistlichen schrift darf dies nicht auffallen. Einige andre stellen, welche schon Mätzner angeführt hat, beweisen, dass der verfasser ein Engländer war, ebenso dass die schwestern englisch als ihre muttersprache betrachteten. ${ }^{68}$ )

Wie jetzt die frage steht, dürfen wir also die Ancren riwle als ein neuangelsächsisches originalwerk ansehen.

Bei bestimmung des ortes, wo das werk entstanden sei, hält Morton sich an die angabe des Oxford codex, dass die schwestern, fur welche es geschrieben, in 'Tarente gelebt hätten. Dies würde also auf Dorset weisen. Die sprache der Cotton mss. stimmt damit vollig uberein. Doch ist dabei nicht aus dem auge za lassen, dass sich die angabe von Tarente neben der sicher falschen findet, Simon von Gent sei der verfasser der lateinischen urschrift. Grosses gewieht diurfen wir also auch nicht auf den namen Tarente legen.

Morton knlipft nnn weiter an den namen eine geistreiche vermutung: $\mathrm{Zu}$ Tarente am Stoure in Nord-Dorset errichtete Ralph de Kahaines, dessen vater mit Wilhelm dem crobrer gekommen war, ein kloster für nonnen, welches Maria und allen

67) So pag. 50. Ich write muchel vor oðre and noðing ne etrineð ou, mine leove sustren, pag. 192 . ze, mine leove sustren. beod peo ancren pet ich iknowe, pet habbed lest neode to uroure azean peos temptaciuns: bute one sicnesse. Vor mid more eise, ne mid more menke, not ich non ancre pet habbe al pet hire neod is pene ze preo habbed: ure loverd beo hit idoncked.

68) Vgl. Mätzner a. a. o. Es sind dies vor allen pag. 172 u. pag. 244. 
heiligen gewidmet war. ${ }^{69}$ ) Dies kloster zerfiel, doch Richard Poor richtete es von neuem ein. Poor war su Tarente geboren, wurde decan zu Chichester in Sussex, dann bischof zu Salisbury und Durham. Zuletzt ging er wider nach Tarente und starb daselbst 1237. ${ }^{71}$ ) Wie sehr er das dortige kloster liebte, beweist, dass er dort sein herz beisetzen liess. Diesem Poor schreibt nun Morton die Ancren riwle zu. Wäre der name 'Tarente besser beglaubigt, so dürften wir unbedingt dieser schönen vermutung beistimmen, allein ihn nennt unter den sieben hss. der nonnenregel nur eine einzige und zwar eine, die, wie Morton selbst gezeigt hat, durch und durch schlecht ist.

IX. Poema morale. ${ }^{71}$ )

So nennen die englischen herausgeber ein in langen reimpaaren geschriebnes gedicht, welches tuber vergänglichkeit des lebens, über tod und jungstes gericht handelt. Es umfasst 198 reimpaare. Es beginnt:

Ich em nu alder pene ich wes a wintre and a lare

Ich welde mare pene ich dede mi wit ahte bon mare,

Well longe ich habbe child ibon a worde and a dede pah ich bo a wintre ald, to zung ich em on rede. Hss. davon gibt es folgende: 1. Egerton ms. 613. Hiernach gab es Furnivall heraus. 2. Lambeth 487 fol. $59 \mathrm{~b}-65 \mathrm{~b}$. Wanley sagt, ${ }^{72}$ ) der schluss sei in prosa (Morris bemerkt hiertiber gar nichts), doch sieht man genauer $z u$, so ist das angebliche prosastilck vers 262-271. Hiermit bricht Lambeth cod. ab, Morris druckte das ende nach dem Egerton ms. ${ }^{73}$ ) 3. Digby ms. No. 4. ${ }^{74}$ ) Hiernach druckte Hickes es ab I, 222. 4. ms. Trinity Coll. Cambridge. Abgesehen von kleinen abweichungen stimmt es mit dem Egerton ms. ${ }^{75}$ )

6!) Schon Morton zeigt, wie Wanleys angabe bei Hickes bd. III, 149 11. a. o., die schwestern hätten dem St. Jakobsorden angehört, auf misverstïndnis einer stelle (pag. 8 bei Morton) beruhe. Morton pag. X. ff.

io) Morton pag. XII-XV.

${ }^{71}$ Abgedruckt ist das gedicht bei Furnivall: Early English poems and lives of Saints. Berlin u. London 1862. - Morris a. a. o. nach Lambeth, ergäinzt durch Egerton ms. - Hickes hat es ebenfalls.

72) Hickes III, bd. 268 .

i3) Vgl. Morris a. a. o. VI oben.

74) Hickes III, p. $\$ 3$.

i) Ebend. p. 169. 
Wir gehen nun zu den homilien, den paternoster, glaubensbekenntnissen, gebeten und ähnl. über, welche in nags. sprache geschrieben sind. Oft ist es allerdings recht schwer zu entscheiden, ob ein denkmal nags. oder altags. sei. Abgesehen davon, dass überhaupt beides sich berührt, gibt es viele hss., welche altags. abgefasst waren, aber von nags. schreibern abgeschrieben wurden. Diese änderten manche alte formen, andre liessen sie stehen. Ein beispiel dafür ist Bodl. cod. F 4, 12. ${ }^{\text {i6}}$ ) Neben gewende, geræd, gelæde findet sich ihaten, isceop, ilomlice, istreona. Sonst wird schon vielfach gekürzt und geschwächt, neben godspel findet sich gospel, mæden (virgo), cnihtes neben cnihtas, brucen, habben, libben (vivere), buten statt butan, wundre für wundra, auch schon übergang der starken declination zur schwachen: mid eadmodre stefnen; rixeð mid alle his halgan. Auch schon die ächt nags. schreibweise dæize neben dxge ist hier anzutreffen. Ebenso zeigt diese mischung ein cod. der bibl. S. James, Westminster. ${ }^{i 7}$ ) Hier steht neben dagas ein bispelles, weorces, neben gen. suna auch sune, die infin. beclyppen, unterstanden neben solchen auf -an. mid felzenden tacnen, on boken u. ähnl. So mag es sich auch mit cod. Hatton. 65 der Bodl. verhalten, welcher angeblich die vier evangelien in nags. sprache umfassen soll. ${ }^{78}$ ) Die proben bei Wanley sind \%u kurz, um darnach etwas entscheiden zu können.

In der ubersicht der nags. homilien sind hier die bereits gedruckten vorangestellt.

X. Hali Meidenhad. ${ }^{79}$ )

"Es ist dies eine homilie uber Ps. XIV, 11: Audi filia et inclina aurem tuam etc. Die hss., welche uns erhalten, sind: Titus D. XVIII, ${ }^{80}$ ) und Bodl. No. 34. ${ }^{81}$ )

XI. De octo viciis et de duodecim abusivis hujus seculi. ${ }^{32}$ ) - Diese abhandlung ist nach einer altags. verfasst,

76) Hickes bd. III, 15-26.

77) Ebend. 181.

78) Vgl. ebend. p. 76.

$\left.{ }^{79}\right) \mathrm{Hg}$. von Cockayne, Hali Maidenhad nach der'Titus hs. Den titel hat Cockayne erfunden.

80) Hickes III, 247.

${ }^{81}$ ) Ebend. p. 80.

82) Morris a. a. o. pag. 101-119. 


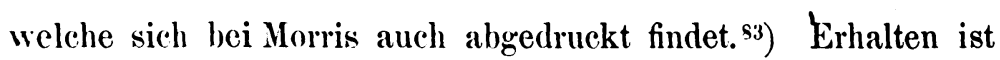
die nags. schrift in Lambeth ms. 487.84)

XII. Sawles warde ${ }^{55}$ ) oder, wie Cockayne es nach den anfangsworten nemnt: Si sciret paterfamilias. Drei hss. sind davon bekannt: Bodl. ms. No. $34 .{ }^{36}$ ) Es beginnt: fol. $75^{\text {b }}$ : I pe feaderes and $\mathrm{i}$ pe sones and $\mathrm{i}$ pe hali gastes nome: her begrinneð sawles ward. Si sciret paterfamilias qua hora fur venturus esset vigilaret utique et non sineret perfodi domum suam. Der schluss dieser hs. fehlt von fol. $84 \mathrm{ab}$. Diese hs. hat Morris seinem drucke zu grunde gelegt. - Den schluss gibt er nach der hs. Reg. ms. 17 A XXVII. ${ }^{87}$ ) - Eine dritte hs. ist ('otton. 'Titus D 18. ${ }^{89}$ ) Diese hs. stimmt abgesehen von mundartlichen abweichungen mit den andern tiberein. Sie ist vollständig, nur fehlt natiirlich der schreibervers am schlusse.

XIII. pe wohunge of ure laverd. ${ }^{89}$ ) Davon ist nur die hs. Titus D XVIII bekannt. Hickes gibt schon nachricht dariber. ${ }^{90}$ )

XIV. Gebete an Jesus.

Unter XIX und XXI hat Morris zwei gebete ${ }^{91}$ ) an (hristus abgedruckt. XIX nach Lambeth $487 ;^{92}$ ) XXI nach Nero A XIV. ${ }^{93}$ ) Abgesehen rom anfange ist in beiden hss. dasselbe gebet. Doch ist XXI vollständiger, als XIX. Pag. 203 z. 10 les druckes bricht Lambeth ms. ab. Doch Nero ms. ist auch nicht vollständig. Der schluss fehlt. - Unter XXIII finden wir bei Morris ein andres gebet an Christus nach Nero A XIV fol. 128"-131 a abgedruckt. "94)

*:3) Eliend. pag. 296-305.

*I) Hickes bil. III, 267 .

8.5) Morris 1). 245-26i7.

*ii) Hickes III, - $\rightarrow$.

${ }^{*}$ ) Vgl. Morris 265 z. 23.

$\left.{ }^{*}\right)$ Hickes III, 247.

s:!) Morris 269-2st.

(*i) Hickes III, 247.

:1) Pag. 16:3 u. pag. 200.

:2) Vgl. Hickes III, 265.

:a) Eluencl. 225. - Planta a. a. 0. 205.

9) Ebend. Wanley teilt dieses gebet nochmals in zwei, indem er lei der stelle: Swete softe iesu iseli beod pet pe luvieð (Morris $215 \mathrm{z.21}$ ) en neues gehet annimmt. 
XV. Marienlied. ${ }^{95}$ )

Es ist in alliteration und mit reimen oder assonanzen geschrieben. So der anfang:

Cristes milde moder seynte marie,

Mines lives leome mi leove lefdi,

To pe ich buwe and mine kneon ich beze

And al min heorte blod to pe ich offrie

pu ert mire soule liht and mine heorte blisse

Mi lif and mi tohope min heale mid iwisse.

Die einzig bekannte hs. dieses liedes ist Cotton. Nero A XIV.96) XVI. Gebet an Maria. ${ }^{97}$ )

Zwei hss. sind uns erhalten. Eine ist die schon oft erwähnte Royal Ms. 17 A 27. Doch ist hier nur ein bruchstiick, welches schliesst: ant te unwurde wohes pat he for us sunfule willeliche. ${ }^{98}$ ) Vollständig findet sich das Gebet Cott. Nero A XIV. ${ }^{99}$ )

XVII. Pater noster in reimen. ${ }^{100}$ )

Es steht im erwähnten Lambeth ms. fol $21^{\mathrm{b}}-25^{\mathrm{a}}$. Der anfang lautet: ${ }^{101}$ )

Pater noster qui es in celis etc.

Ure feder pet in heovene is, pet is al soð ful iwis.

XVIII. Pater noster ${ }^{102}$ ) und andere glaubensformeln.

Es ist von Wright nach einer hs. Caius College zu Cambridge gedruckt.

XIX. Credo.

Eines fuhrt Wanley an, welches wol in diese zeit zu setzen ist. ${ }^{103}$ ) Es beginnt: Credo in deum ic bileve on God patrem omnipotentem pene almihti fader und schliesst: po gode on

95) Morris a. a. o. pag. 191-200.

$\left.{ }^{96}\right)$ Hickes III, p. 228.

97) Morris pag. 205-208 nach Nero.

98) Abgedruckt ist das bruchstiick Morr. pag. 305. Verglichen mit der Cott. hs. bricht es pag. 207 z. 2 bei Morr. ab.

99) Hickes III, 228.

${ }^{100)}$ Morris pag. 55-72.

101) Hickes III, 267.

102) Reliquiae antiquae ed. by Wright and Halliwell I, 2\$2.

${ }^{103)}$ Hickes III, 169. Vgl. jedoch auch das anm. 122 gesagte. 
eche hlisse and wele on ure loverd Jhu Criste on hevene. pe uvele on eche wope and pine mid pe devel on elle sowle and licam abuten ende. Ein anderes glaubensbekenntnis enthält Cotton. ms. Nero A XIV. ${ }^{104}$ ) Darnach hat es Morris ahgedruckt. ${ }^{105}$ ) Das credo und paternoster, welches in den reliquiae antiquae mitgeteilt wird, ${ }^{106}$ ) ist schon dem altenglischen zuzuteilen.

XX. De initio creature. ${ }^{i 07}$ )

So nennt Morris sehr wenig passend eine predigt iber den sündenfall der engel und menschen und die erlösung der kinder Adams durch Christi tod, höllenfahrt und auforstehung. Erhalten ist. uns dies denkmal in mss. Cott. Vesp. A 22 108) fol. $54-56^{b}$.

XXI. Parabel vom reichen könige. ${ }^{109}$ )

Morris gibt dieser predigt den titel: an bispel. Sie findet sich im selben cod. wie die vorige, fol. $56^{\mathrm{b}}-58^{\mathrm{b}}$.

XXII. Induite vos armatura dei. ${ }^{110}$ )

Die homilie beginnt: Ur hlaford sanctes paulus pe is peoden lareaw us maned and menezed of sume wepne to nemene pus ewedende: Induite vos armatura dei. - Auch dieses werk steht im angeführten Cott. ms. 58 . - Diese homilie ist jedenfalls unvollständig. Wanley hat dies richtig eingesehen, Morris hält sie fïr vollständig. "11) Jedenfalls hat Wanley recht, denn die homilie endet: pu ahst to habben ehte wopnocin, pa beod sceold, helm and brenie, swrd and spere, stede and twe $i$ sporen and ane smearte zerd. Hwic scule beon ure sceld? sanctus paulus hus seið: In omnibus sumentes scutum fidei, in quo possitis omnia tela nequissimi (ignea) extinguere. Hiermit kamn dic homilie nicht geschlossen haben.

101) Hickes bd. III 2.24.

10i) Morris pag. 217.

106) Rel. antiquae I pag. 57 nach Brit. Mus. Harl. 3724.

107) Morris $217-231$. Es ist diese homilie wahrscheinlich nach einer homilie Aelficics angefertigt, die betitelt wird: Sermo de initio creaturae al populum. In so fern ist der gegebne name einigermassen gerechttertigt.

108) Hickes 111, 242.

109) Morris pag. 231-241.

${ }^{110)}$ Morris pag. 241-243. Der text findet sich Eph. VI, 11.

iii) Hickes III, 242. 
XXIII. Erant appropinquantes. ${ }^{112}$ )

Es ist diese homilie wahrscheinlich nach einer von Älfric gearbeitet "Dominica IV post pentecosten “. Die nags. homilie findet sich ebenfalls Vespasian A 22. Wanley erwähnt sie nicht. limam.

XXIV. Quum appropinquasset ihesus iereso-

Von dieser homilie besitzen wir zwei bearbeitungen. Sie ist fur den palmsonntag geschrieben. Die eine version findet sich Lambeth ms. 487. ${ }^{113}$ ) Hiernach hat sie Morris gedruckt. ${ }^{114}$ ) Eine andre homilie steht Otho A 13.115) Sie beginnt: Ure drihten nechlende his passiun isohte preo stedes pider ward and pet was pe feorde. Schluss: pet he lede ey on domes dæi into hewenriche pe o schal ileste. Quae nobis prestet qui secla per omnia regnat.

XXV. Ecce nunc tempus acceptabile, ecce nunc dies salutis. ${ }^{116}$ )

Auch hiertiber sind uns zwei nags. homilien erhalten. Die eine findet sich Trin. Coll. Cambr. ${ }^{117}$ ) und fängt an: pe hevenliche leche seinte Poul nimed zeme of ure sawle sicnesse pet ben ure sinnes ponzed wurse him and minezer us bi his holie write. Schluss: and overcumen at ende hem and alle pe ping pæt us to sunne te $\partial$. Quod ipse prestare dignetur q. v. etc. Die andre bearbeitung ist im Lamb. ms., wonach Morris sie veröffentlicht hat.

XXVI. In leinten time uwile mon gar to scrifte. ${ }^{118}$ )

Nur im Lambeth ms. uns erhalten.

XXVII. In diebus dominicis. ${ }^{119}$ )

Diese betrachtung tuber die heiligkeit des sonntages hat uns auch nur das Lambeth ms. uberliefert.

142) Morris a. a. o. pag. 243-245. Wir haben hier, wie bei der vorigen, nur ein bruchstück vor uns.

113) Hickes III, 266.

114) Morris 1-11.

115) Hickes III, 233.

11i) Morris 11-25 trägt die uiberschrift: Hic dicendum est de quadragesima.

117) Hickes III, 170.

118) Morris 25-41.

119) Ebend. $41-47$. 


\section{Missus est Jeremias in puteum etc. ${ }^{12 "}$ )}

Ebenfalls nur in der Lambeth hs.

XXIX. 'l'ria sunt hominum saluti necessaria, fides, baptismus, nundicia vite. IDiese erklärung des glaubensbekenntnisses ist im Lambeth ms. unvollstïndig. ${ }^{121}$ ) Morris bemerkt zwar nichts dariber, doch ist es ganz klar. Eine zweite hs. Trinit. Coll. geht viel weiter und bietet wahrscheinlich einen vollständigen text. ${ }^{122}$ )

Endlich gibt es noch eine hs. Otho A 13, welche vielleicht dieselbe abhandlung, wenn auch etwas abweichend, enthält. ${ }^{123}$ ) Leider ist aus Wanleys diurftigen angaben nichts $\mathrm{zu}$ sehen. Sie beginnt: Unicuique hominum ete. Bifore alle ping preo ping beod efric man helwurpe and erest bihoved to habbe. Diese homilie ist jedenfalls vollständig, denn sie schliesst: pet we mote eche riche habbe o o bute ende. Qui vivit ete. Leicht können gelehrte in England feststellen, wie sich diese homilie zu den zwei andern verhält.

\section{Vom barmherzigen Samariter. ${ }^{124}$ )}

Dies stiick beginnt: Homo quidam descendebat ab ierusalem in ierico ete. Nur im Lambeth cod. ist es uns erhalten. ${ }^{125}$ )

$\left.{ }^{120}\right)$ Ebend. 47-54, iiberschrift: Hic dicendum est de propheta.

121) Morris nach Lambeth, also jedenfalls nach der unvollkommensten hs., pag. 73 -7s. - Hickes III, 169.

12:2) Vollstïndig ist der text, wenn wir die drei von Wanley unter $V-V I I$ incl. gegebenen stiicke $z u$ e in er homilie verbinden. No. VI das credo gehört jedenfalls zu V und entspricht Morris p. 75 z. $25 \mathrm{ff}$. Es schliesst: pe gode on eche blisse and wele mid ure loverd Jhu Criste on hevene. pe uvele on eche wope and pine mid pe devel, on eche (so ist wol statt Wanleys ..elle" zu lesen) sowle and licam abuten ende. Hiermit ist jedenfalls die erkliirung des credo heendet, doch hat sich sehr wahrscheinlich die von Wanley unter VII gegehene erklïrung des paternoster daran geschlossen. Anfang: Pater noster etc. pu singest pe salm pe me clepeð crede pu seist pæt on gode bilevest and dost cnovnesse pæet he is liverd, ac panne pu singest pe salm pat is cleped pater noster. Schluss: Ac lese us loferl of his ezzinge and of alle uvele. Amen. Swo hi: wurde. - Hiermit scheint diese homilie geschlossen zu haben. Diese meinung bestiitigt auch der umstand, lass nun sowol im Lambeth cud. als im Trin. Coll. codex „in die natalis Domini“ folgt.

12:3) Ehend. 23:3.

124) Morris hat es unter der wenig bezeichnenden benennung: ..De natale lomini" pag. 79-86 abgedruckt.

${ }^{125)}$ Hickes III, $26 \%$. 
XXXI. Pfingstpredig t. ${ }^{126}$ )

Nur im Lambeth cod. ${ }^{127}$ ) Sie fängt an: fram pan halie hester dei bod italde fifti daza to pisse deie and pes dei is ihaten pentecostes.

XXXII. Factus est filius dei omnibus sibi obtemperantibus causa salutis eterne. ${ }^{128}$ )

Diese homilie ist nach Älfric gearbeitet. ${ }^{129}$ ) Sie steht im Lambetlı cod.

XXXIII. Christus passus est pro nobis, relinquens exemplum. ${ }^{130}$ )

Auch diese betrachtung ist nur in Lambeth.

XXXIV. Qui parce seminat parce et metet. ${ }^{131}$ )

Diese homilie findet sich unter der bezeichnung: de Sancto Laurentio im ms. Trin. Coll. Cambr. ${ }^{132}$ ) Eine andere hs. ist der Lambeth cod., wonach Morris die schrift veröfientlicht hat. ${ }^{133}$ ) Der anfang beider hiss. stimmt ziemlich iberein, das ende hingegen geht auseinander.

XXXV. Reverenda est nobis hec dies sancta que dicitur dominica.134)

Ausser dem gegenstande hat diese homilie nichts mit XXVII gemein. Zwei hss. sind uns erhalten: 1. Lambeth ms. 2. Cott. Otho A 13. ${ }^{135}$ ) Die anfünge stimmen fast wörtlich, der schluss der Cott. hs. lautet: pider mote we cume par is wane of alle ifel and fille of iche gode. Lambeth hat hier noch latein eingemischt.

XXXVI. Qui vult venire post me abneget semet ipsum etc. ${ }^{136}$ )

Diese abhandlung wird bewahrt im Lambeth ms. und in

126) Morris gibt sie unter: in die pentecosten pag. $87--101$.

127) Hickes III, 267.

128) Morris 119-125 als: dominica $\mathrm{V}$ quadragesimac.

129) Morris XI.

$\left.{ }^{130}\right)$ Ebend. pag. 125-131 als: dominica II post pascha.

$\left.{ }^{131}\right)$ Morris pag. 131-139.

132) Hickes 171.

133) Hickes 268.

134) Morris p. 139-145.

135) Hickes 233.

136) Morris p. 145-150. - Hickes 172. 
oben erwähnten ms. 'Trin. Coll. Cambr. Beide mss. stimmen zienlich mit einander. Endlich im Otho A 13. ${ }^{137}$ )

XXXVII. Estote fortes in bello et pugnate cum antiquo serpente. ${ }^{135}$ )

Drei hss. dieser homilie sind bekannt: 1. 'Trin. Coll. ('ambr. ${ }^{139}$ ) 2. Lambeth ms. 3. Cott. Otho $A$ 13.140) Die letzte hs. hat den schluss etwas verändert: Mirl pis wepne was David iscryd po po he Goliam his unwine ofer com, two wile God pat we ure mohte.

XXXVIII. Euntes ibant et flebant. ${ }^{1+1}$ )

Im Otho A $13 \mathrm{~ms}$. führt diese homilie den titel: "Sermo de jacobo apostolo ". 142) Ebenfalls ist diese schrift auch im Lambeth ms. erhalten. Beide hss. stimmen ziemlich mit einander. Eine dritte hs. ist die des 'Trin. Coll., die dem Cott. zunächst steht.

XXXIX. Maria virgo assumpta est ad ethereum thalamum. ${ }^{143}$ )

Diese homilie ist uns nur in dem schon of erwähnten Cambr. cod. Trin. Coll. B, 14, 52 erhalten. ${ }^{144}$ )

XL. Nox precessit, dies autem appropinquabit. ${ }^{145}$ )

Der Trin. cod. bringt diese abhandlung. ${ }^{146}$ )

Hiermit ist die zahl der in zugänglichen drucken verötfientlichten nags. denkmäler geschlossen. Es folgen nun die unedierten. Für die homilien sind zwei hss. wichtig. Die erste ist Cambr. cod. B. 14. Diese will Morris fur die Early 'T.Soc. herausgreben. ${ }^{147}$ ) Die andre ist eine Cottoniana, Otho $\mathrm{A}, 13 .{ }^{149}$ )

137) Als sermo de martiribus bezeichnet (Otho diese homilie (vgl. Hickes 233). ('ambr. ms. stimmt genau mit Lambeth.

134) Morris 151-157.

13:) Hickes 172. 140) Ebend. 2:3:3.

141) Ebend. pag. 155-15\%.

14:) Hickes 23:3.

143) Rel. antiqu. bd. I pag. 1:28.

144) Hickes III, 171.

145) Rel. ant. ebend. pag. 130 betitelt: Dominica tertia.

14i) Hickes III, 169.

147) Vgl. Morris XV. Es sollen die homilien der Cambr. hs. den :Weiten teil der ..Old English homilies" bilden. Nach dem ...Eigth report of the Committee, January $1572 *$ pag. 6 ist das werk, welches durchaus rotwendig fiir die kenntnis des nags. ist, fast fertig gedruckt.

14k) Hickes III, 2:33. 
Das Cambridge ms. enthält ausser den schon angeftihrten $\left.{ }^{149}\right)$ :

XLI. Apparuerunt apostolis dispartite lingue tanquam ignis. ${ }^{150}$ ) Der anfang lautet:

pope ure loverd Jhu Crist fundede lichamliche fro eorde (Wanley: ecede) to hevene he forbed his ap. and hire holi ferreden poet hie neren noht sorie.

XIII. Ambulans Jhe juxta mare Galilee. ${ }^{151}$ )

Anfang: pe holi godspel of pis dei spect of ure helend and of two brodren, peet on is S. P. and paet oder S. Andrew and seið pat ure helende giede bi pe se.

XLIII. Convertimini ad me in toto corde vestro. ${ }^{152}$ )

Anfang: Non eoroliche fader ne moder haved sva mildeheorte to hire life child swo ure hevenliche fadr haved to us. ponked wurde him.

XLIV. Cum immundus 'spiritus ab homine exierit, vadit per loca arida. ${ }^{153}$ )

pe loverd Seint Matteu speed on his holi godspel of pe grimliche wordes pe ure hælend at sume time zaf to andswere pe unbilefile Iudeuische men.

XLV. Dominus de celo prospexit super filios hominum. ${ }^{154}$ )

pe holi prophet Davio seid on ane stede on pe salmboc pe wordes pe ich her nu seide. per he speed of pe mildhertnesse pe ure loverd Jhu Crist doð men.

XIVI. Ecee venit rex, occuramus obviam salvatori nostro. $\left.{ }^{15 \pi}\right)$

'To dai is cumen pe holie tid pet me elepep advent, panked be ure loverd Ihu Crist pit haved isend and hit lasted pre wuke fulle and sum del more.

199) Ebend. pag. 169-172.

150) Ebend. 171 mit der bezeichnung: in die pentecostes.

151) Ebend. iiberschrieben: de sancto andrea.

152) Ebend. 170 bezeichnet: in capite ieiunii.

153) Ebend. betitelt: in media Xea.

154) Hickes III, 171.

155) Ebend. 169. Es ist dort bezeichnet: „de adventu“. 
XLVII. Ego vox clamantis in deserto, parate viam domini. ${ }^{156}$ )

pe loverd Seint lucas guittinned(?) on his pe wunderliche hiderkume and pe corolich herbiwist and pe wunderliche heöen sid of ure loverd Seint Johan Baptiste.

XLVIII. Egredietur virga de radice Jesse. ${ }^{157}$ )

An zerd sal spruten of Jesse more and an blosme stien of pare more and uppe pare blosme resten pe holie gost.

IXL. Elevatus est sol in celum. ${ }^{159}$ )

pe holi prophete Abacue pe wunede (W. wundede) on pis weorlde and eft per of wot fele hundresl wintre er pe time pe ure drihten unterstod manisshe.

L. Estote prudentes et vigilate in orationibus. ${ }^{159}$ )

pe hevenliche keyhirde Seinte peter iseih pret ure eldren hadden fele fon and we habbed alswo pe ben al to snelle (W. smiele) on swikedom.

LI. Hæe est dies quam fecit dominus, exultemus et letemur in ea. ${ }^{160}$ )

pis dei haveð ure drihten makeð to gladien and to blissen us. ponked wuròe lim.

LII. Hora est jam nos de sompno surgere. ${ }^{161}$ )

pe laverd seinte Powel pe is heved-lorpeov (W. Lordeau) of alle, holie chirechen bihicld pis wrech woreld and sazh pæt mast mannen ladden hire lif on simnen and pret hem likede here lodliche sinnes.

LIII. Inter natos mulierum non surrexit major Johanne Baptista. ${ }^{162}$ )

An lizer man ofte ligeð and a soðsage man seið ofte so $\varnothing$. pe nevre ne lihgh ne lige ne wite ne ne mai pat is ure hælende.

LIV. Libera nos domine de morte eterna. ${ }^{163}$ )

pe lifholi man Job pe pe boe of speed and seið: erat vir ille simplex et justus ete.

15ti) Ebend. 171, betitelt: de Sco Johanne Baptista.

15i) Ebend. 172, ohne titel.

$\left.{ }^{15 k}\right)$ p. 171, benannt: In ascensione domini.

1.5) p. 172. titel fehlt.

(fiv) p. 170 bezeichnet: In die pasche.

(ii)) p. 169 als Dominica II in adventu.

$\left.{ }^{162}\right)$ p. 171 ohne bezeichnung.

1iii) Hickes 1II, 171 ist iiberschrieben: de defunctis. 
LV. Natus est nobis salvator qui est Christus. ${ }^{164}$ )

Gode tioinge and murie to heren us tellè pe loverd seinte lucas on pe holie godspelle.

LVI. Mulier que erat in civitate nomine Marie jam penitens. ${ }^{165}$ )

pe' laverd S. L, pe irenned pret holi godspel pe men ræd in holie chiriche seið par on.

LVII. Obtulerunt pro eo domino par turturarum. ${ }^{166}$ )

To dai man mai iheren he pe wile wich peaw wes on pe olde laze mid wimmen on pre pinges, pret on is childbed, and pæt oðer chirchganz and pe pridde pe offrinz.

LVIII. Omne datum optimum et omne donum perfectum desursum est. ${ }^{167}$ )

Seint jacob pe holie apostel pe ure drihten sette to lorpeove pe folc of Irslm. he nam zeme of pe wune pe weren po.

LIX. Posuerant peccatores laqueum mihi. ${ }^{168}$ )

pe sinfulle haved leid zarne me to henten and ich ne forlet pine bode. Ure fo fared and hunted and leid zarne in a wildernesse to henten pe deor pe wunied per inne.

LX. Preoccupemus faciem domini et in psalmis jubilemus ei. ${ }^{169}$ )

pe holie prophete Davið munezeð us on pe sealmboc to berewen pisse ( $W$. berezentis pe) wile pe we muzen.

LXI. Quomodo cantabimus canticum domini in terra aliena. ${ }^{170}$ )

pe holie prophete David specp on pe sealmboc and on a stede peron munezep sume of pe wordes pe weren spoken bitwinne two folkes.

LXII. Reges 'Tharsis et insule munera offerent, reges Arabum et Sabæ dona adducent. ${ }^{171}$ )

164) Ebend. 170 als: in die natali domini.

165) Führt den titel: Maria Magdalena. Hickes 171.

$\left.{ }^{166}\right)$ Ebend. 170 ist sie benannt: purificatio Mariae.

${ }^{167)}$ Ebend. 171.

168) Ebend. 172.

169) Hickes III, 170 ohne titel.

170) Ebend.

iit) Ehend. als ..de epiphania* bezeichnet. 
Nid mede man mai over water faren and mid weldede of give frend wuerthe.

LXIII. Stetit Jesus in medio discipulorum suorum. ${ }^{172}$ )

We reden on pe holi godspel-boc pret ure helende provede on pe holi rode and deatie polede and mid his edeliche deað̇e lesde us of deatie.

LXIV. 'Turbe que precedebant Dominum et que sequebantur elamabant dicentes: Osanna. ${ }^{173}$ )

It is custume pret ech chirchsocne goo pis dei a procession

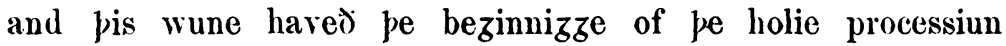
pe ure helende makede toward te stede per he wolde ded polen.

Otho A 13 enthält folgende homilien:

LXV. Apparuit beni'gnitas et humanitas. ${ }^{174}$ )

Iwrn us was gesvuteled purh vitegie and erendrache pat god wolde man bimmeal for ure sache.

LXVI. Cum natus esset Jesus in Beethlehem Jude. $\left.{ }^{175}\right)$

God almihti pe his fader and sune and holi gost on preo wise to dai his michele mihte swetelede.

LXVII. Ego sum panis vivus. ${ }^{176}$ )

Ure drilten spech on one stede on pan holie godspelle.

LXVIII. Intravit Jesus in quoddam eastellum. ${ }^{177}$ )

Lucas pe godspellere pe trehnede pe holie gadspel of pisse dei

LXIX. Mulierem fornicantem recepi. ${ }^{178}$ )

Ure drihte pe zef boxe pe olde laze and pe newe. 1)as verhältnis dieser homilie zu LVI ist noch zu untersuchen. LXX. Que est ista que ascendit. ${ }^{179}$ )

pe hevenliche ewen ure lefedi Seinte marie heveð tif feste imne twelf monar.

Mit der ersten bei Wright und Halliwell abgedruckten

172) Diese homilie fiihrt weiter keinen titel.

$\left.{ }^{173}\right)$ Hickes 170, bezeichnet: Dominica Palmarum.

ii) Ebend. 2:33, ,in die natali domini“ iiberschrieben.

17i) Hickes III, 233. Als „sermo in epiphania domini“ aufgefübrt.

176) Ebend. ohne titel. _ ${ }^{177}$ ) Ebenfalls ohne titel.

${ }^{178)}$ Sermo de Maria Magdalena benannt.

${ }^{179}$ ) Als sermo beate virginis von Wanley angegeben. 
homilie (vgl. oben XXXIX) hat hier diese nichts zu tun, wenn auch sie sich im inhalte nahe beruhren.

Hiermit schliessen wir die übersicht der nags. denkmäler.

Wanley führt noch einige mehr an. Doch wie es sich mit der nags. evangelienübersetzung verhält, haben wir oben pag. 76 gesehen. Ein angeblich lateinisch-neuags. glossar ist erst noch zu untersuchen, in welche zeit es gehört. Wanley sagt nur, ${ }^{180}$ ) es sei ${ }^{2}$ ante quingentos annos" geschrieben.

Schliesslich führt Wanley ${ }^{181}$ ) noch aus Cott. Calig. A IX, also aus derselben hs., welche die hs. A des Lazamon und eine hs. der eule und nachtigal enthält, ein gedicht an: „de morte, judicio et de poenis infernis" als nags. Es begiunt:

Non mai longe lives wene

Ac ofte him lico pe wrench,

Feir weðer turneð oft into reine

And wunderliche hit make $\delta$ his blench ete.

Madden setzt dieses mss. bekanntlich in die erste hälfte des 13. jh. Doch dürfen wir es dann gewis nicht viel vor 1250 entstanden denken. Denn das gedicht zeigt keine eutschieden alte formen, ausserdem ist die reimstellung:

$$
\text { a } b \text { a b b a } a \text { b }
$$

doch schon eine sehr künstliche, während sonst nags. nur paarweise gereimt wird. Besser wird es also in spätere zeit zu setzen sein.

Die neue ausgabe von Warton's literaturgeschichte (ed. by Hazlitt, London 1871,4 voll.) wurde bei unsrer darstellung noch nicht benutzt. Doch gibt dieselbe auch nichts neues. Nur ist das in anm. 6 gesagte teilweise zuriickzunehmen, indem viel verbesserungen jetzt in den text aufgenommen sind. - Die denkmäler, welche Craik (a compendious history of English literature from the Norman conquest, 2 voll.) anfihrt pag. $193 \mathrm{ff}$., aus Ritsons anc. songs, also das lied von ('anut, die w'orte Aldreds u.a.sind hier absichtlich iibergangen, weil sie sehr zweifelhafter natur sind. Nur die Godric zugeschriebenen zeilen dürften noch hierher gehören.

Nachdem ferner die neue ausgabe von Mätzners Englischer grammatik erschienen, ist das in anm. 14 gesagte etwas zu modificiren, vgl. Mätzner 2. aufl. I. teil, 1. abt. pag. 7 oben.

180) Hickes III, 84, ms. der Bodleiana. - ${ }^{181}$ ) Hickes III, 229.

I.EIPZIG. RICHARD WÜLCKER. 